\title{
Efficacy of different-frequency TEAS on acute pain after the total knee arthroplasty: a study protocol for a parallel group randomized trial
}

Yulin Li $i^{1}$, Lixi Chu', Xiangming $\mathrm{Li}^{2}$, Weitao Zhai, ${ }^{3,4}$, Yinghui $\mathrm{Ma}^{3,4}$, Yong $\mathrm{He}^{3,4}$, Yuelin $\mathrm{Xu}^{3,4}$, Sheng Ding ${ }^{3,4}$, Huali Gao ${ }^{3,4}$, Jing Zhang ${ }^{3,4}$, Bowen $\mathrm{Ye}^{3,4}$, Jingchao Wang ${ }^{3,4}$, Jie Yao ${ }^{3,4}$, Chonggui Wu ${ }^{3,4}$ and Lianbo Xiao ${ }^{3^{*}}$

\begin{abstract}
Background: Total knee arthroplasty (TKA) is an optimal option for patients with middle-to-end-stage knee osteoarthritis. However, the management of postoperative acute pain remains inefficient. Transcutaneous electrical acupoint stimulation (TEAS) is a nonpharmacological method to manage postoperative acute pain. Different frequencies of TEAS have been tested using varying parameters, but the optimal analgesic frequency remains controversial. The aim of this study was to explore the optimal analgesic frequency of TEAS for treating acute pain after the primary unilateral TKA.

Methods/design: This is a double-blind, randomized controlled trial. A total of 156 patients are randomly assigned to: G1, $5 \mathrm{~Hz}$ TEAS; G2, $100 \mathrm{~Hz}$ TEAS; G3, mixed TEAS (alternative use of daily $5 \mathrm{~Hz}$ and $100 \mathrm{~Hz}$ TEAS) and G4, placebo TEAS. In the G1, G2 and G3 groups, TEAS is conducted at acupoints SP9 and GB34 of the leg that was operated on (at a wave of continuous, balanced and asymmetrical biphasic square, with a pulse width of $200 \mu \mathrm{s}$, and a strong but comfortable current) for 30 min prior to a 30-min rehabilitation session per day for 2 weeks. In G4 group, TEAS is delivered at a strong but comfortable current for $30 \mathrm{~s}$, then the current is gradually decreased to none over the next $15 \mathrm{~s}$. The primary outcomes are measured before surgery, at baseline (POD 3, before TEAS intervention), week 1 and 2 after TEAS intervention with the Numeric Pain Rating Scale and The American Knee Society Score. The secondary outcomes include: (1) Active range of motion of the knee that was operated on; (2) Surface electromyography of both quadriceps; (3) Modified 30-s sit to stand test; (4) Additional usage of analgesia; and (5) SF-36. The additional outcomes include: (1) Patients' satisfaction rate; (2) Patient's expectation rate; and (3) Incidence of analgesia-related side effects. To test the blinding of participants and assessors, they are asked to guess whether the subjects received active or placebo TEAS within 5 min after the latest intervention. The safety and financial cost of TEAS are assessed.
\end{abstract}

Discussion: Mixed TEAS has more favorable effect on acute pain control than the placebo or $5 \mathrm{~Hz}$ or $100 \mathrm{~Hz}$ TEAS.

Trial registration: ChiCTR1800016347. Date of registration was 26 May 2018. Retrospectively registered.

\footnotetext{
*Correspondence: 13701888178@163.com

${ }^{3}$ Guanghua Hospital of Integrated Traditional Chinese Medicine and Western

Medicine, Shanghai 200000, China

Full list of author information is available at the end of the article
}

(c) The Author(s). 2019 Open Access This article is distributed under the terms of the Creative Commons Attribution 4.0 International License (http://creativecommons.org/licenses/by/4.0/), which permits unrestricted use, distribution, and reproduction in any medium, provided you give appropriate credit to the original author(s) and the source, provide a link to the Creative Commons license, and indicate if changes were made. The Creative Commons Public Domain Dedication waiver (http://creativecommons.org/publicdomain/zero/1.0/) applies to the data made available in this article, unless otherwise stated. 


\section{Background}

\section{The need for total knee arthroplasty}

Osteoarthritis of the hip and knee ranks 11th among the 291 causes of disability, and 38th highest among disabilityadjusted life years globally [1]. The age-standardized prevalence of global knee osteoarthritis (KOA) is 3.8\%, higher in elderly women $[1,2]$. The optimal treatment for middle-toend-stage KOA is total knee arthroplasty (TKA) [1, 2] which can relieve pain, recover function and improve early stage quality of life (QoL) of KOA patients [2, 3]. The therapeutic methods for middle-to-end-stage KOA have been gradually redefined, TKA of which will remain at hand in the next few decades [4]. In 2012, more than 670,000 cases of TKA were accomplished in the USA alone [5], 94 to $97 \%$ of which were operated on for KOA [2].

\section{Acute pain after the TKA}

Surgery is the second most common cause of persistent pain [6]. Though half of all TKA patients report postoperative pain relief [7], 44\% of them still suffer continued pain at 3-4 years after the operation [6]. Inadequate post-TKA pain control can lead to adverse events [8], like knee stiffness, infection, deep venous thrombosis $[5,9]$, and undermine the patients' ability to perform physical activities, like stair-climbing and heavy manual work [10]. The post-TKA pain also reduces the patients' satisfaction with this surgery [11] in as many as $18.2 \%$ [12]. It can be said that recently developed interventional and preventive strategies have all failed to effectively control the post-TKA pain [13].

\section{Side effects of analgesic drugs}

There are multiple ways to control post-TKA pain [14]. "The panel recommends that clinicians offer multimodal analgesia...combined with nonpharmacological interventions, for the treatment of postoperative pain...(strong recommendation, high-quality evidence)" [7]. In clinical practice, surgeons and anesthesiologists usually seek help from opioids [15]. But opioids, taken either before or after the surgery, always produce side effects that can disable physical activities and lower patient satisfaction. Patients with a history of chronic opioid use who successfully decreased their intake of opioids before TKA had substantially improved clinical outcomes that were comparable to patients who did not use opioids at all [16]. At postoperative day (POD) 1 after TKA, at least $25 \%$ of patients showed an Opioid-Related Symptom Distress Scale score $>1$ for nausea, drowsiness, itchiness and fatigue $[15,17]$. Long-term postoperative use of opioids may lead to opioid-induced hyperalgesia $(\mathrm{OIH})$, a paradoxical response to opioids $[13,18,19]$. OIH may reduce opioid efficacy and exacerbate the pain [16].

\section{Advantages of transcutaneous electrical acupoint stimulation}

Given the side effects of opioids, the nonpharmacological approach has been developed as an adjuvant therapy to reduce pain. Transcutaneous electrical nerve stimulation (TENS) is an economical and non-invasive technique $[20,21]$ with few side effects $[14,22]$. TENS with electrodes placed on various acupoints is also called transcutaneous electrical acupoint stimulation (TEAS) [23]. Compared with conventional acupuncture, TEAS brings no risk of needle-induced infection and is more acceptable for those fearing needle stimulation [24]. Evidence has proven that TEAS can effectively reduce postoperative acute pain, the consumption of opioids and opioid-related side effects, and improve patient satisfaction, physical function, and promote the enhanced recovery after surgery (ERAS) [15, 25, 26].

\section{Objectives}

To evaluate the efficacy of different frequencies of TEAS on acute pain after the TKA.

\section{Methods/design}

A mono-center, prospective, randomized, parallel-controlled, participants-, assessor- and statistician-blinded study will be carried out to find the optimal analgesic frequency of TEAS by comparing the efficacy of different frequency on postoperative acute pain control in patients undergoing the primary unilateral TKA. This study strictly followed the principles of the Declaration of Helsinki. The study protocol had been approved by the Clinical Trial Ethics Committee of the Shanghai Guanghua Hospital of Integrated Traditional Chinese and Western Medicine (approval number: 2017-k-41-01) and registered at the Chinese Clinical Trial Registry (http:// www.chictr.org.cn/listbycreater.aspx, ChiCTR1800016347). The researchers will be trained in the consistency of the symptoms and signs, the evaluation process.

\section{Eligibility criteria}

The inclusion criteria: (1) 50-80 years old; (2) diagnosis of KOA; (3) prepared to undergo primary unilateral TKA; (4) treated with the same TKA procedures; (5) be American Society of Anesthesiologists (ASA) grades I-II; (6) treated with standard general anesthesia. The exclusion criteria: (1) hyposensitivity or impairment in the leg that was operated on; (2) having used opioids for more than 2 weeks within the preoperative 6 months; (3) having received other nonpharmacological treatments within the preoperative 2 weeks that might affect the outcomes; and (4) showing TENS contraindications, including the use of pacemakers or being allergic to nickel. 


\section{Sample size}

The sample size will be calculated using pain ratings from the previous studies [27-29]. The formula:

$$
n=\frac{2\left(Z_{\alpha}+Z_{1-\beta}\right)^{2} \sigma^{2}}{\Delta^{2}}
$$

An effect size $(\Delta)$ of 2 with a standard deviation (SD) of 2.88 between low-frequency (LF) TEAS and placeboTEAS $(n=33 \times 4 / 0.85=156)$, an $\Delta$ of 2.35 with a SD of 2.56 between high-frequency (HF) TEAS and placeboTEAS $(n=19 \times 4 / 0.85=95)$, an $\Delta$ of 1.1 with a SD of 0.7 between mixed TEAS and placebo-TEAS $(n=7 \times 4 / 0.85$ =35) [26]. Using this information, a four-group study will be established: 33 patients in each group, $\alpha=0.05$ (two-sided), $\beta=0.2$ ( $80 \%$ power) [30]. Considering a possible sample loss of $15 \%$, the final sample size will be set with 39 patients in each group.

\section{Recruitment strategies and enrollment}

KOA inpatients who are scheduled to undergo a primary unilateral TKA between May 2018 to May 2019 will be recruited by the Department of Joint Surgery, the Shanghai Guanghua Hospital of Integrated Traditional Chinese and Western Medicine. The main recruiting mode will be propagated by a resident doctor $(\mathrm{A})$. The KOA inpatients willing to participate in our study will be screened by specific researchers (B). The inpatients who meet the screening criteria will be clearly aware of their involvement in this study. The participant who needs to be transferred to the intensive care unit after a standard TKA procedure or perioperative interference will be excluded. These participants often have some complications of other organ systems, including cardiopulmonary insufficiency or damaged liver or kidney function, which may interference the baseline characteristics of the participant of this clinical trial. Those participants who transfer to the general ward will be asked to provide informed consent.

Figure 1 shows the trial flow chart for the patient screening, treatment allocation, intervention, outcomes assessment and data analysis. Figure 2 provides an overview of the study conduct, review, description and interpretation. The populated Standard Protocol Items: Recommendations for Interventional Trials (SPIRIT) Checklist is presented in Additional file 1.

\section{Randomisation and allocation concealment}

Participants who meet the screening criteria will be randomized into four balanced groups: G1, G2, G3 and G4. The randomization sequence will be computer-generated by an independent research staff $(\mathrm{C})$ with a 1:1:1:1 ratio using SPSS 21.0.

At POD 3 and before the TEAS application, three physical therapists $(\mathrm{D}, \mathrm{E}, \mathrm{F})$ will be mainly responsible for the treatment sessions and outcomes evaluation.

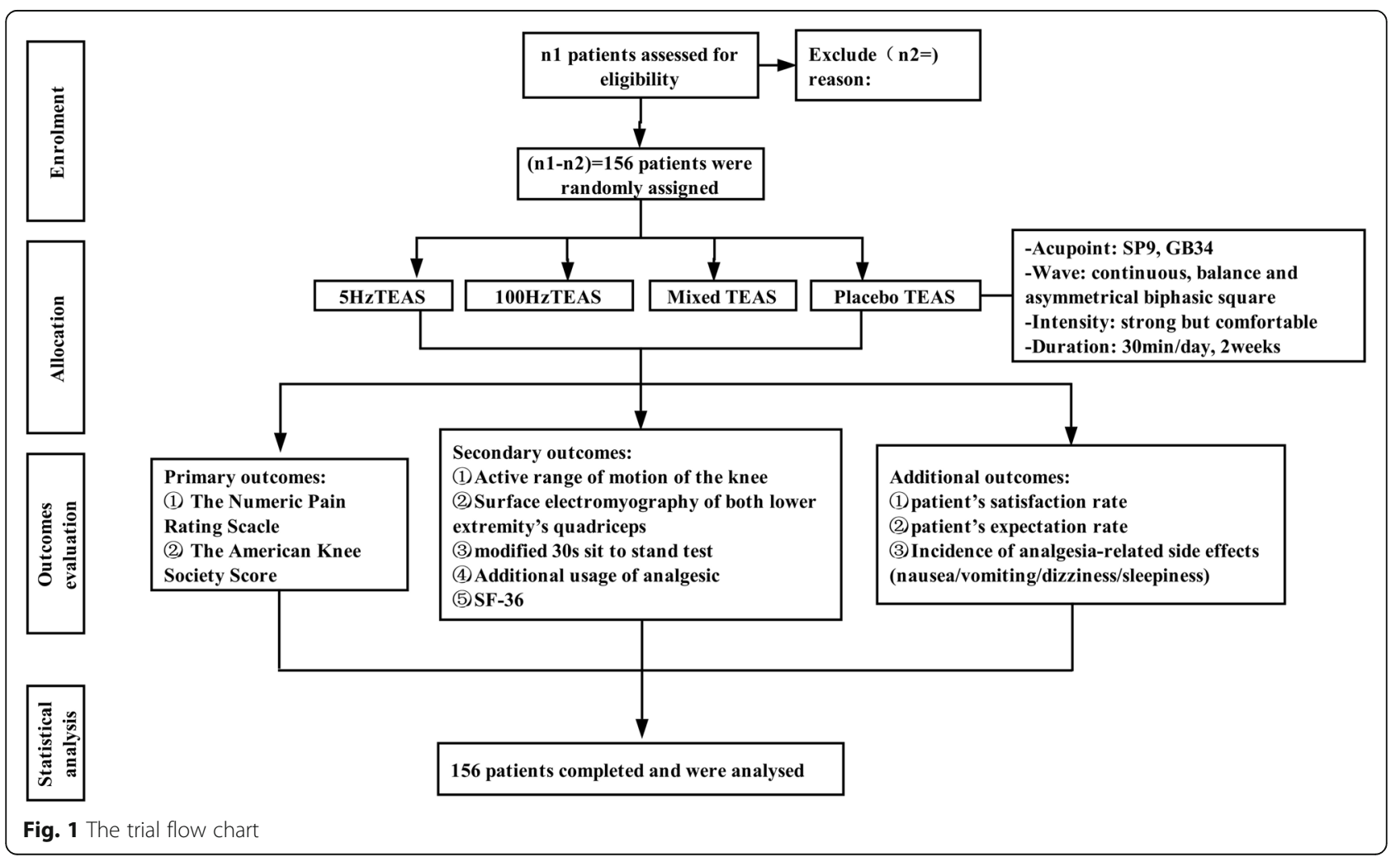




\begin{tabular}{|c|c|c|c|c|c|c|c|c|c|c|c|c|c|c|c|}
\hline & \multirow{3}{*}{ TIMEPOINT } & \multicolumn{14}{|c|}{ Study Period } \\
\hline & & \multirow[t]{2}{*}{ Enrollment } & \multirow[t]{2}{*}{ Allocation } & \multirow{2}{*}{$\begin{array}{c}\text { Pre-op* } \\
1 \text { day }\end{array}$} & \multirow{2}{*}{$\begin{array}{c}\text { POD } \\
3\end{array}$} & \multicolumn{5}{|c|}{$\begin{array}{l}\text { The 1st week of } \\
\text { TEAS intervention }\end{array}$} & \multicolumn{5}{|c|}{$\begin{array}{l}\text { The 2nd week of } \\
\text { TEAS intervention }\end{array}$} \\
\hline & & & & & & 1 & 2 & 3 & 4 & 5 & 1 & 2 & 3 & 4 & 5 \\
\hline \multicolumn{2}{|r|}{ Eligibility screen } & $\mathrm{x}$ & & & & & & & & & & & & & \\
\hline \multicolumn{2}{|c|}{ Demographic information Informed } & $\mathrm{x}$ & & & & & & & & & & & & & \\
\hline \multicolumn{2}{|c|}{ Medical information } & $\mathrm{x}$ & & & & & & & & & & & & & \\
\hline \multicolumn{2}{|r|}{ Allocation } & & $\mathrm{x}$ & & & & & & & & & & & & \\
\hline \multicolumn{2}{|r|}{ consent } & & & & $\mathrm{x}$ & & & & & & & & & & \\
\hline \multirow{2}{*}{$\begin{array}{l}\text { Primary } \\
\text { outcomes }\end{array}$} & NRS & & & 4 & 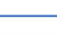 & - & 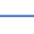 & & - & - & & 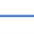 & & & $\rightarrow$ \\
\hline & AKSS & & & $\mathrm{x}$ & $\mathrm{x}$ & & & & & $\mathrm{x}$ & & & & & $\mathrm{x}$ \\
\hline \multirow{6}{*}{$\begin{array}{l}\text { Secondary } \\
\text { outcomes }\end{array}$} & AROM of the operated & & & & & & & & & & & & & & \\
\hline & knee & & & 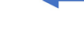 & & & & & & 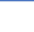 & & & & & $\Rightarrow$ \\
\hline & sEMG of both quadriceps & & & $\mathrm{x}$ & $\mathrm{x}$ & & & & & $\mathrm{x}$ & & & & & $\mathrm{x}$ \\
\hline & $\mathrm{m} 30 \mathrm{STS}$ test & & & $\mathrm{x}$ & $\mathrm{x}$ & & & & & $\mathrm{x}$ & & & & & $\mathrm{x}$ \\
\hline & Additional usage of & & & & & $<$ & & & & & & & & & $\rightarrow$ \\
\hline & & & & & & & & & & & & & & & \\
\hline \multirow{5}{*}{$\begin{array}{l}\text { Additional } \\
\text { outcomes }\end{array}$} & Patients' satisfaction rate & & & & & $\mathrm{x}$ & & & & & & & & & \\
\hline & patient's expectation rate & & & & & $\mathrm{x}$ & & & & & & & & & \\
\hline & Incidence of analgesia- & & & & 4 & & & & & & & & & & $\rightarrow$ \\
\hline & related side effects ** & & & & 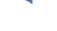 & & & & & & & & & & 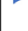 \\
\hline & SF-36 & & & & $\mathrm{x}$ & & & & & & & & & & $\mathrm{x}$ \\
\hline \multicolumn{2}{|c|}{ Adverse events of TEAS } & & & & & 4 & - & - & - & 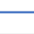 & 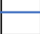 & 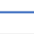 & - & 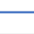 & $\rightarrow$ \\
\hline \multicolumn{2}{|c|}{ Assess the blinding*** } & & & & & & & & & & & & & & $\mathrm{x}$ \\
\hline
\end{tabular}

Fig. 2 The schedule of trial enrollment, interventions and assessments

Initially, D will perform the basic measurements of participants in four groups and double-check whether the sensation in the leg that was operated on is normal. After D leaves the ward, E will apply a 30-min TEAS intervention and subsequently $\mathrm{F}$ will perform a 30 -min rehabilitation session per day in four groups. One TEAS intervention and one rehabilitation constitute one treatment session. Immediately after the end of one treatment session, D will return to the ward, evaluate and record the outcomes according to the trial design. Researchers except for $\mathrm{C}$ and $\mathrm{E}$ will be blinded to the treatment allocation. Randomisation and allocation concealment in a light-tight sealed envelope until $\mathrm{E}$ firstly reveals the TEAS intervention according to the random number sequence at POD 3. Participants will be required not to communicate with any other researchers, participants and care providers to minimize the risk of potential bias. After the latest TEAS intervention, participants and outcome assessors will be asked to guess whether the subjects received active TEAS or placebo TEAS within $5 \mathrm{~min}$ to evaluate the blinding quality.

\section{Interventions}

Scheme of standard analgesic use

The scheme of standard analgesics used for each participant includes two parts. First, patient-controlled analgesia (PCA) will be used from the day of surgery to the postoperative day (POD) 3, and in the meanwhile, flurbiprofen axetil (100 $\mathrm{mg})$ by intravenous drip is to be administered twice a day. Second, during the POD 4 to POD 14, the participants will only intake etocoxib orally, $60 \mathrm{mg}$ qid.

All participants can ask for additional analgesia if necessary. The additional consumption of analgesia (type and dosage) will be recorded daily and finally statistically analyzed.

\section{TEAS interventions}

TEAS will be performed using $\mathrm{XCH}-\mathrm{C} 1$ equipment (ShangHai Nuo Cheng Co.). Based on the theory of Traditional Chinese Medicine, two circular electrodes (2 $\mathrm{cm} \times 2 \mathrm{~cm}$ ) will be placed around the surgical knee acupoints of SP9 (YINLINGQUAN) and GB34 (YANGLINGQUAN). SP9 is located at the hollow between 
the inferior edge of the medial tibial condyle and the medial edge of the tibia. GB34 is located at the lateral side of the calf in the hollow, anterior and inferior to the fibular head [31]. Before electrode attachment, E will shave and sterilize the skin with $75 \%$ ethyl alcohol. The TEAS produces a balanced, asymmetrical biphasic waveform with a pulse width of $200 \mu \mathrm{s}$, and a strong but comfortable current amplitude [32]. Intervention duration will be set as 30 min per day for 2 weeks [33]. Participants will be required not to change the current settings by themselves during the application of TEAS. A 30-min TEAS will be daily applied prior to a 30-min rehabilitation (including flexibility, strength, range of motion, balance and gait exercises [34]).

For the active TEAS $(5 \mathrm{~Hz} / 100 \mathrm{~Hz} /$ mixed $)$ group, a continuous frequency of $5 \mathrm{~Hz} / 100 \mathrm{~Hz} /$ mixed will be used. The placebo group will deliver a strong but comfortable current for $30 \mathrm{~s}$ which then gradually vanishes over the next $15 \mathrm{~s}$. One study [35] has demonstrated that this new sham device can promote the effect of blinding through eliminating expectation bias and clarifying the true efficacy of TEAS. In this way, a sound emitted at the end of TEAS will indicate the endpoint of the TEAS treatment, which achieves the best blinding effect on participants and the outcomes assessor. Meanwhile, the participant will be told that: the TEAS works all the time during its application and they may or may not experience sensations from the TEAS unit due to different sensation thresholds of different people.

\section{Outcomes evaluations}

Pain intensity (rest and movement) and the American Knee Society Score (AKSS) are the primary outcomes. Secondary outcomes include active range of motion (AROM) of the knee that was operated on, surface electromyography of both quadriceps, the modified 30-s sit to stand test, additional use of analgesics and the 36-item Short Form health survey (SF-36). The additional outcomes include patient satisfaction rate, patient expectation rate and the incidence of analgesia-related side effects.

The pain intensity, AROM, incidences of analgesia-related and TEAS-related side effects will be daily recorded. Evaluation of the patient satisfaction rate and patient expectation rate will be conducted after the first episode of the TEAS intervention. The SF-36 will be evaluated at two time points: before the TEAS intervention, and 2 weeks after the TEAS intervention. The assessment time points of the remaining outcomes are before the surgery, after the surgery before the TEAS intervention(at POD 3), and 1 week and 2 weeks after the TEAS intervention.

\section{Demographic and medical variables}

Preoperative demographic information includes sex, age, height, weight, duration of KOA, type and duration of complication, marital status, occupation, racial background, education level, preoperative medication and history of major operations.

\section{Pain intensity (resting and movement)}

Pain intensity is usually measured based on an 11-point Numerical Rating Scale (0-10 NRS) [36, 37]. The NRS is a horizontal line marked with the numbers $0-10$ at equal intervals where 0 indicates "no pain" and 10 indicates "worst pain imaginable." The NRS is the preferred intensity scale rather than Verbal Descriptor Scale, and Visual Analog Scale with a lower error rate and standard validity [38]. Most importantly, attributes of the NRS are not related to patient age.

Pain intensity evaluation will be carried out at rest and with movement. Prior to other procedures, the pain intensity at rest is evaluated in a position where the participant lies comfortably and still. The pain intensity at the maximum active flexion of the knee that was operated on is recorded as the pain intensity with movement.

\section{The American Knee Society Score (AKSS)}

The AKSS is commonly used for outcomes evaluation after the TKA globally [39], including knee assessment $(0-100)$ and functional assessment $(0-100)$, a total of 200 points [40]. The knee assessment consist of three components: pain $(0-50)$, range of motion $(0-25)$ and stability $(0-25)$. Deduction is determined by the presence and severity of flexion contracture (0-15), extension lag (EL, 0$15)$ and malalignment $(0-20)$. Function is evaluated via walking $(0-50)$ and climbing stairs $(0-50)$ with deductions for walking aid (0-20). For each part, a negative score is possible and should be transformed to zero.

The knee stability evaluation consists of two parts: the anterior-posterior stability test and the lateral stability test. The procedure of the anterior-posterior stability test is the same as that of the anterior drawer test of the knee. During the lateral stability test, the surgical knee is flexed by $30^{\circ}$. The participant will be required to stand when measuring the lower extremity alignment, and the acute angle between two lines (anterior superior iliac spine to the midpoint of the knee, the midpoint of the knee to the midpoint of the ankle) will be recorded. Valgus of $5-10^{\circ}$ is considered to be the normal alignment and three scores are deducted for each degree of malalignment outside this range [41]. For measuring EL, a towel roll will be placed under the heel of the knee that was operated on. The participant will be required to extend their knee as much as possible, and keep the knee as straight as possible to raise the lower extremity off the towel roll. The physical therapist keeps the goniometer in alignment to measure the degrees from the straight position. The number of degrees is subtracted from the knee extension AROM to obtain the degrees of EL [42]. 


\section{AROM measurement of the operated knee}

In order to reduce the measurement error to the greatest extent, the knee AROM will be measured using a standard goniometer by the same physical therapist [43]. Bone marks of the great trochanter, the lateral epicondyle of the femur and the lateral malleolus will be firstly identified. The axis of the goniometer is positioned over the center of the lateral epicondyle of the femur. The distal arm is aligned with the fibula using the lateral malleolus and the proximal fixed arm is aligned with the femur using the greater trochanter. During the measurement, the participants will be asked to flex or extend the knee that was operated on to the maximal extent of pain that they could tolerate. Supine position is adopted for measuring the knee AROM. During the measurement of the AROM of knee flexion, participants will be required to slide the heel towards the buttocks by themselves to the greatest extent possible. When measuring the measurement of the operated knee (AROM) of knee extension, participants will be asked to extend the knee where a towel roll is placed under the heel as straight as possible. A positive value indicates a flexion position when the initial position of the knee is at the maximum extension position. AROM will be measured in triplicate for calculating the average AROM value.

\section{Modified 30-s sit to stand (m30STS) test}

The m30STS test will be conducted to assess the physical performance of the elderly, ensuring that people with lower body functions can also complete the test [44].

Before the m30STS test, the evaluator should first clearly introduce it to the participants. A trial is needed prior to the real test. The participant will be required to sit in the middle of the standard armchair (17 in. in height and 18 in. in width), back straight, feet shoulder-width apart, and placed on the floor at an angle slightly back from the knee. Instructions to the participate are: "When I say ' $1,2,3$ start', you stand up and then sit down again with both your lower limbs together as much as possible. Try your best to stand up and sit down as many times as possible until the end of $30 \mathrm{~s}$. You can use your hands to help you stand up or sit down if necessary, and I will stand by your side to protect you." The evaluator will record the number of STS repetitions.

\section{Surface electromyographic (sEMG) of both quadriceps}

Surface EMG will be measured simultaneously with the m30STS test. The sEMG data will be collected using a TeleMyo 2400 wireless sEMG tester (Noraxon, USA) for three major muscles of both leg: vastus medialis (VM), rectus femoris (RF) and vastus lateralis (VL), with six tunnels in total, and $1500 \mathrm{~Hz}$ of the sampling frequency. The electrode is a disposable ECG electrode $(\mathrm{Ag} / \mathrm{Cl}, \mathrm{T}-800)$.
Participants will be requested to wear loose pants to expose the thigh prior to sEMG. For securing the quality of the sEMG signal, the evaluator should shave the skin, sterilized with $75 \%$ alcohol and allow it to evaporate before attachment of the electrodes. Bipolar electrodes must be placed parallel to the estimated longitudinal axis of the muscle fibers below, including RF (the midpoint between the anterior superior iliac spine and the upper margin of the patella), VM (the evaluator places a towel roll under the non-surgical knee's popliteal fossa and asks the participant to extend the knee to press the towel roll forcefully, then the bulging area slightly proximal and medial to the patella is the muscle belly of VM. Since the quadriceps of the knee that was operated on after surgery are so weak making finding the muscle belly difficult, the same area of the non-surgical knee symmetrical to the operated one, therefore, will be selected to perform the test) and VL (the midpoint between the greater trochanter and the inferior margin of the patella). The center-to-center electrode distance is 2 $\mathrm{cm}$. The reference electrode is placed over the fibula head. For fastening the electrodes, the evaluator will use athletic tape for adding security without additional pressure on the top of the electrodes. Finally, fastening the wires against the body will prevent the electrodes from being pulled off, but the wires will not be placed in a way that alters the movement patterns of the participants.

A pressure transducer, instead of a high-speed camera, will be used to objectively identify the time point. The seventh tunnel (the type of tunnel is "test TTL") will be connected to the FSR400 force-sensitive resistance transducer (the sensitivity ranges from 0 to $10 \mathrm{~kg}$ ) to measure the change of the buttocks' pressure during the m30STS test. The pressure signals can be transported simultaneously to the computer by a mainboard (Arduino UNO). The start time point of standing up (t1) and the end time point of sitting down ( $\mathrm{t} 2$ ) will be precisely identified once the pressure value is obtained by the A0 port (range 0-1023) of mainboard exceeds 200. Data exported will be analyzed by Matlab programming. Firstly, the sEMG signals exported in the middle three STS repetitions will be denoised using a $10-400-\mathrm{Hz}$ bandpass filter. The duration ( $\mathrm{t} 2-\mathrm{t} 1)$ of one STS test will be standardized by unifying interpolation of 10,000 data points. Root mean square (RMS), peak value and integrated EMG (iEMG) will be finally calculated after duration standardization. Each index will be tested three times to record the average value for statistic analysis. An engineer blinded to treatment allocation will conduct a sEMG analysis.

\section{SF-36}

Assessment of musculoskeletal disease consists of two parts: the expected outcome of a particular treatment and the effect of a particular treatment on the function, 
QoL and satisfaction of participants. The combination of general instruments and specific questionnaires may be the best way to evaluate the surgical outcomes, since misinterpretations and conclusions with limited reliability can be efficiently avoided [45]. In this study, the specific AKSS questionnaire and the generic instrument of the SF-36 will be utilized.

The SF-36 consists of eight scaled scores covering 36 items: vitality, physical functioning, bodily pain, general health perceptions, physical role functioning, emotional role functioning, social role functioning and mental health. Each scale independently produces the score from 0 to 100 and the lower score indicates a worse disability.

\section{The additional outcomes}

Both physical factors and psychological factors contribute to dissatisfaction [46]. Hence, the patient satisfaction rate or expectation rate will be used to control the psychological factors. Participants will be asked to rank their satisfaction with a 5 -point scale $(1=$ very unsatisfactory; 2 = unsatisfactory; $3=$ neutral; $4=$ satisfactory; 5 = very satisfactory), and the frequency of the "very satisfactory" determines the satisfactory ratio [47]. Patient's expectation rating is ranked with 3 -point scale $(1=$ positive; $2=$ neutral; $3=$ negative). The incidence of side effects of analgesia (nausea/vomiting/dizziness/sleepiness) and the TEAS (local irritation, redness, contact dermatitis, or muscle ache) will be recorded daily.

\section{Statistical analysis}

All data will be analyzed using the statistical package for IBM SPSS Statistics V21. The intention-to-treat (ITT) and per-protocol sets (PP) will be analyzed. Continuous data are presented as mean \pm standard deviation (SD), and categorical variables are described as percentages. Baseline data will be collected and analyzed. Continuous data with normal distribution and homogeneity variance will be analyzed using analysis of variance (ANOVA). Otherwise, the Kruskal-Wallis $H$ test will be performed.

Statistical analysis includes the following four aspects. Firstly, the repeated measurement data will be analyzed using the mixed-effects model or generalized estimating equation (GEE) or generalized linear mixed models (GLMMs). Secondly, the correlation between different outcomes will be analyzed using the multiple linear regression or logistic regression. In this study, NRS, sEMG or AROM are independent variables, whereas the AKSS or m30STS test are dependent variables. Psychological factors serve as covariance. Thirdly, the safety analysis of TEAS will be compared between groups using $X^{2}$ test or Fisher's exact test. Fourthly, health economic evaluation (cost-effectiveness analysis) will be performed. A $P$ value $<0.05$ (two-side) is considered statistically significant.

\section{Discussion}

The management of postoperative acute pain remains a challenge. In clinical practice, opioids are essential in the management of postoperative acute pain although multiple opioid-sparing approaches have been developed [48]. However, opioid-related side effects markedly limit therapeutic efficacy and patient satisfaction. Thus, nonpharmacological therapies adjunct to the multimodal analgesic regimen are urgently required [49]. TEAS, a nonpharmacological and noninvasive treatment, is commonly used for the management of postoperative acute pain with fewer adverse effects.

The frequency of TEAS varies from low $(\mathrm{LF}<10 \mathrm{~Hz})$ to high frequency $(\mathrm{HF}>50 \mathrm{~Hz})$ [21]. The gate control theory and endogenous opioids mechanism are involved in TEAS [20]. The gate control theory explains the analgesic effect of HF stimulation. Compared to LF, HF is more effective in selectively activating large-diameter $A \beta$ afferent sensory fibers and $\delta$ opioid receptors in the spinal cord [50] without simultaneously exciting small-diameter $\mathrm{C}$ fibers to reduce the transmission of noxious stimuli. HF TEAS exerts a segmental analgesic effect on localizing the specific dermatome with a rapid onset and offset. LF stimulation can be explained by the endogenous-opioid mechanism. LF activates endogenous opioids through stimulating small-diameter $A \delta$ afferent sensory fibers and $\mu$ opioid receptors (MORs) [50]. Subsequently, endogenous opiates are released to provide the pain control effect. The analgesic effect of LF is extrasegmental, delayed but lasting. However, repeated electrical stimulation can also lead to opioid tolerance and cross-tolerance equivalent to repeated administration of $\mu$ or $\delta$ opioid receptor agonists [51]. The separated application of TEAS at $5 \mathrm{~Hz}$ and $100 \mathrm{~Hz}$ at alternative days may delay the tolerance effect [52, 53].

The frequency of TEAS is a key parameter for the effective management of postoperative acute pain control, while the optimal frequency remains controversial.

In vivo studies $[50,54]$ have suggested that both LF and HF electrical stimulation provide analgesic effect and strengthen the endogenous analgesic system in experimental animals without side effects. But Xiang et al. [55] revealed that $2-\mathrm{Hz}$ TEAS but not $100-\mathrm{Hz}$ TEAS induced an upregulation of MOR binding potential in numerous pain-related brain areas, and such effects even last until the end of the 2-Hz TEAS intervention in some brain areas. Yu et al. [24] yielded identical results in a rat model that shows that LF TEAS efficiently alleviated the neuropathic pain by regulating the positive expression of MORs in the dorsal root ganglion. In a clinical research, conducted by Huang et al. [26], mixed TEAS considerably reduced the demand for opioids and relieved postoperative 
acute pain compared to $2 \mathrm{~Hz}$ and $100 \mathrm{~Hz}$ TEAS alone. One hundred $\mathrm{Hz}$ TEAS can decrease the incidence of postoperative nausea and vomiting in patients undergoing video-assisted thoracic surgery. Tokuda et al. [56] also found that compared to a placebo group and a control group, the modulated-frequency electrical stimulation group had a lower pain score at rest and with movement, and better pulmonary function. However, they did not compare curative effect between modulated-frequency and LF or HF electrical stimulation. Desantana et al. [29] and Pitangui et al. [57] demonstrated that both HF and LF stimulation considerably reduced the intensity of postoperative pain compared to the placebo group. The analgesic effects between the LF and HF group was not compared. However, Oliveira et al. [56] concluded that different frequencies $(0 \mathrm{~Hz}, 7 \mathrm{~Hz}, 100 \mathrm{~Hz}$ and $255 \mathrm{~Hz})$ of electrical stimulation applied to $\mathrm{C} 6-\mathrm{C} 8$ did not show considerable change in the pressure-induced pain threshold or cold-stimulation-induced discomfort in healthy adults. A Cochrane review reported by Walsh et al. [58] in 2011 demonstrated that it is unable to make any definitive conclusions about the effectiveness of electrical stimulation as an isolated treatment for acute pain in adults. Johnson et al. [59] updated this Cochrane review in 2015 by adding another seven studies. They provided preliminary evidence that active electrical stimulation could decrease the acute pain in adults compared to sham electrical stimulation, but this conclusion may come with a high risk of bias because of inappropriate research design. Consistent with previous research, Bjordal [60] found that electrical stimulation notably decreased the demand for postoperative analgesics. However, a system review oppositely demonstrated that HF stimulation was effective in pain control and whether LF stimulation was better than standard care after TKA remains controversial [61]. Another system review obtained an opposite conclusion that the frequency of electrical stimulation had no effect on hypoalgesia [62].

We speculated that inappropriate research design and TEAS parameter selection may explain these controversial conclusions among previous studies. Lack of description of treatment allocation, insufficient blindness control or inappropriate sham method may significantly discount research credibility [59, 63-65]. Besides, inappropriate selection of TEAS parameters may lead to poor quality of the TEAS intervention, thus affecting the efficacy of postoperative acute pain management [61, 65]. Selection of intervention parameters of TEAS is decisive in nonpharmacological therapies [65].

In this study, the research design and selection of TEAS parameters will be modified. The sample size, admission time balance and blinding effect are strictly controlled [35]. In the previous studies aiming to find the optimal frequency, $2 \mathrm{~Hz}, 4 \mathrm{~Hz}$ or $5 \mathrm{~Hz}$ were frequently used for investigating the analgesic effect of low frequency, and 80 $\mathrm{Hz}$ or $100 \mathrm{~Hz}$ were frequently used for investigating the analgesic effect of high frequency $[26,29,51,55,66]$. The purpose of this clinical trial is to evaluate the different analgesic effects of low frequency, high frequency and mixed frequency on acute pain after the TKA, rather than evaluating different analgesic effects between different frequency parameter inside the high frequency or low frequency. So we will choose $5 \mathrm{~Hz}$ as a representative of low frequency, and $100 \mathrm{~Hz}$ as a representative of high frequency. A strong but comfortable current amplitude reported by the participant will be selected to achieve the best analgesic effects [32]. An intervention duration of 30 to $40 \mathrm{~min}$ was demanded for the complete expression of pain control effect [33]. The only variable factor of this study is the frequency of TEAS, so the non-research factors which may interfere with the outcomes are well controlled. Thus, stimulation parameters including a strong but comfortable current amplitude, 30-min intervention duration and $200-\mu$ s pulse width are consistent in all groups.

Therefore, we hypothesize that the mixed use of TEAS at different frequencies can improve postoperative acute pain, the muscle performance and knee function.

\section{Additional file}

Additional file 1: SPIRIT 2013 Checklist. (DOC 140 kb)

\section{Abbreviations}

AKSS: The American Knee Society Knee Score; ANOVA: Analysis of variance; AROM: Active range of motion; ASA: The American Society of Anesthesiologists; ERAS: The Enhanced Recovery After Surgery; HF: High frequency; iEMG: Integrated electromyography; ITT: Intention-to-treat analysis; KOA: Knee osteoarthritis; LF: Low frequency; m30STS: Modified 30-s sit to stand test; MOR: $\mu$ opioid receptor; NRS: Numeric rating scale; OA: Osteoarthritis; OlH: Opioid-induced hyperalgesia; ORSDS: Opioid-Related Symptom Distress Scale; POD: Postoperative day; PP: Per-protocol sets analysis; QoL: Quality of life; RF: Rectus femoris; RMS: Root mean square; SD: Standard deviation; sEMG: Surface electromyography;

TEAS: Transcutaneous electrical acupoint stimulation; TENS: Transcutaneous electrical nerve stimulation; TKA: Total knee arthroplasty; VL: Vastus lateralis; VM: Vastus medialis

\section{Acknowledgements}

Not applicable.

\section{Funding}

The Science and Technology Commission of Shanghai Municipality, China.

\section{Availability of data and materials}

The datasets used and/or analysed during the current study are available from the corresponding author on reasonable request.

Trial status

$\checkmark$ Protocol Version number: V.01: 12 October 2018

$\checkmark$ The date recruitment began: 10 May 2018

$\checkmark$ The approximate date that recruitment was completed: 31 May 2018

Authors' contributions

Study sponsor: LBX. Conception, design and supervision of the study: LYL, LBX, LXC. Draft and revision of the manuscript: YLL. Clinical trial registration and ethics approval: YLL. Analysis of sEMG: XML. Statistic analysis: YHM. The 
surgery for TKA: WTZ, YLX, YH, SD, HLG, BWY, JCW. Principle evaluators: JY. The application of TEAS: JZ. Rehabilitation session: CGW. All authors read and approved the final manuscript.

\section{Ethics approval and consent to participate}

The study has been approved by the Ethics Committee of Guanghua Hospital of integrated traditional Chinese and Western Medicine in Shanghai, approval No. of the Ethic Committee: 2017-k-41-01. All participants will sign an informed consent before starting the study.

\section{Consent for publication}

Not applicable.

\section{Competing interests}

The authors declare that they have no competing interests.

\section{Publisher's Note}

Springer Nature remains neutral with regard to jurisdictional claims in published maps and institutional affiliations.

\section{Author details}

${ }^{1}$ Faculty of Rehabilitation Medicine, The Shanghai University of Traditional Chinese Medicine, Shanghai 200000, China. ${ }^{2}$ Department of Orthopaedics, Guanghua Hospital of Integrated Traditional Chinese Medicine and Western Medicine, The Shanghai University of Traditional Chinese Medicine, Shanghai 200000, China. ${ }^{3}$ Guanghua Hospital of Integrated Traditional Chinese Medicine and Western Medicine, Shanghai 200000, China. ${ }^{4}$ Traditional Chinese Medicine and Western Medicine, Shanghai 200000, China.

Received: 14 November 2018 Accepted: 22 April 2019

Published online: 29 May 2019

\section{References}

1. Lalmohamed A, Vestergaard $P, B$ Beer $A D$, et al. Changes in mortality patterns following total hip or knee arthroplasty over the past two decades: a nationwide cohort study. Arthritis Rheumatol. 2014;66(2):311-8 Available from: http://www.ncbi.n/m.nih.gov/pubmed/24504803.

2. Carr AJ, Robertsson O, Graves S, et al. Knee replacement. Lancet. 2012; 379(9823):1331-40.

3. Shan L, Shan B, Suzuki A, et al. Intermediate and long-term quality of life after total knee replacement: a systematic review and meta-analysis. J Bone Jt Surg Am. 2015;97(2):156-68 Available from: http://www.ncbi.nlm.nih.gov/ pubmed/25609443.

4. Maradit KH, Larson DR, Crowson CS, et al. Prevalence of total hip and knee replacement in the United States. J Bone Jt Surg Am. 2015;97(17):1386-97 Available from: http://www.ncbi.nlm.nih.gov/pubmed/26333733.

5. Skou ST, Roos EM, Laursen MB, et al. A randomized, controlled trial of total knee replacement. N Engl J Med. 2015;373:1597-606.

6. Wylde $\mathrm{V}$, Hewlett $\mathrm{S}$, Learmonth ID, et al. Persistent pain after joint replacement: prevalence, sensory qualities, and postoperative determinants. Pain. 2011;152(3):566-72 Available from: http://www.ncbi.nlm.nih.gov/ pubmed/21239114.

7. Chou R, Gordon DB, de Leon-Casasola OA, et al. Management of postoperative pain: a Clinical Practice Guideline from the American Pain Society, the American Society of Regional Anesthesia and Pain Medicine, and the American Society of Anesthesiologists' Committee on Regional Anesthesia, Executive Commi. J Pain. 2016;17(2):131-57 Available from: http://www.ncbi.nlm.nih.gov/pubmed/26827847.

8. Terkawi AS, Mavridis D, Sessler DI, et al. Pain management modalities after total knee arthroplasty: a network meta-analysis of 170 randomized controlled trials. Anesthesiology. 2017;126(5):923-37 Available from: http:// www.ncbi.nlm.nih.gov/pubmed/28288050.

9. Liddle AD, Judge A, Pandit H, Murray DW. Adverse outcomes after total and unicompartmental knee replacement in 101330 matched patients: a study of data from the National Joint Registry for England and Wales. Lancet. 2014;384(9952):1437-45.

10. Smith $T$, Withers $T$, Luben $R$, et al. Changes in physical activity following total hip or knee arthroplasty a matched case-control study from the EPICNorfolk cohort. Clin Rehabil. 2017;31(11):1548-57. Available from: https:// www.ncbi.nlm.nih.gov/pubmed/28438034.
11. Lewis GN, Rice DA, McNair PJ, et al. Predictors of persistent pain after total knee arthroplasty: a systematic reviewand meta-analysis. $\mathrm{Br} J$ Anaesth. 2015;114(4):551-61.

12. Baker PN, van der Meulen JH, Lewsey J, et al. The role of pain and function in determining patient satisfaction after total knee replacement: data from the National Joint Registry for England and Wales. J Bone Jt Surg - Br Vol. 2007;89-B(7):893-900 Available from: http://www.bjj.boneandjoint.org.uk/ cgi/doi/10.1302/0301-620X.89B7.19091.

13. Wu CL, Raja SN. Treatment of acute postoperative pain. Lancet. 2011; 377(9784):2215-25.

14. Johnson MI. Transcutaneous electrical nerve stimulation (TENS) as an adjunct for pain management in perioperative settings: a critical review. Expert Rev Neurother. 2017;17(10):1013-27 Available from: http://www.ncbi. nlm.nih.gov/pubmed/28817978.

15. Tu Q, Gan JH, Shi JL, et al. Effect of transcutaneous electrical acupoint stimulation on postoperative analgesia after ureteroscopic lithotripsy: a randomized controlled trial. Urolithiasis. 2018:1-9.

16. Nguyen LC, Sing DC, Bozic KJ. Preoperative reduction of opioid use before total joint arthroplasty. J Arthroplast. 2016;31(9 Suppl):282-7 Available from: http://www.ncbi.nlm.nih.gov/pubmed/27105557.

17. YaDeau JT, Liu SS, Rade MC, et al. Performance characteristics and validation of the opioid-related symptom distress scale for evaluation of analgesic side effects after orthopedic surgery. Anesth Analg. 2011;113(2):369-77.

18. Angst MS, Clark JD. Opioid-induced hyperalgesia a qualitative systematic review. Anesthesiology. 2006;104(3):570-87.

19. Woolf CJ, Salter MW. Neuronal plasticity: increasing the gain in pain. Science. 2000;288(5472):1765-8. Available from: https://www.ncbi.nlm.nih. gov/pubmed/10846153.

20. Johnson MI. Transcutaneous electrical nerve stimulation (TENS). Wilry; 2012. p. 259-282

21. DeSantana JM, Walsh DM, Vance $C$, et al. Effectiveness of transcutaneous electrical nerve stimulation for treatment of hyperalgesia and pain. Curr Rheumatol Rep. 2008;10:492-9.

22. Li J, Song Y. Transcutaneous electrical nerve stimulation for postoperative pain control after total knee arthroplasty: a meta-analysis of randomized controlled trials. Medicine. 2017;96(37):e8036 Available from: http://www. ncbi.nlm.nih.gov/pubmed/28906393.

23. Ashburn MA, Caplan RA, Carr DB, et al. Practice guidelines for acute pain management in the perioperative setting. Anesthesiology. 2004;100(6): 1573-81 Available from: isi:000221551300032.

24. Yu XD, Zhang FX, Chen BN. Effect of transcutaneous electrical acupuncture point stimulation at different frequencies in a rat model of neuropathic pain; 2016. p. 1-6.

25. Yao YS, Zhao QY, Gong CS, et al. Transcutaneous electrical acupoint stimulation improves the postoperative quality of recovery and analgesia after gynecological laparoscopic surgery: a Rrndomized controlled trial. Evid Based Complement Alternat Med. 2015:1-6.

26. Huang S, Peng WP, Tian $X$, et al. Effects of transcutaneous electrical acupoint stimulation at different frequencies on perioperative anesthetic dosage, recovery, complications, and prognosis in video-assisted thoracic surgical lobectomy: a randomized, double-blinded, placebo-controlled trial. J Anesth. 2017;31(1):58-65.

27. Rakel B, Zimmerman MB, Geasland K, et al. Transcutaneous electrical nerve stimulation for the control of pain during rehabilitation after total knee arthroplasty: a randomized, blinded, placebo-controlled trial. Pain. 2014;155(12): 2599-611 Available from: http://www.ncbi.nlm.nih.gov/pubmed/25270585.

28. Hamza MA, White PF, Ahmed HE, et al. Effect of the frequency of transcutaneous electrical nerve stimulation on the postoperative opioid analgesic requirement and recovery profile. Anesthesiology. 1999;91(5):1232-8.

29. Desantana JM, Sluka KA, Lauretti GR. High and low frequency TENS reduce postoperative pain intensity after laparoscopic tubal ligation: a randomized controlled trial. Clin J Pain. 2009;25(1):12-9. https://doi.org/10.1097/AJP. Ob013e31817d1070.

30. Kadam P, Bhalerao S. Sample size calculation. Int J Ayurveda Res. 2014;118(4):55-7

31. Liang F. Science of acupuncture and moxibustion. 2nd ed. Shanghai: Shanghai Science and Technology Press; 2012. p. 62-105.

32. Pantaleao MA, Laurino MF, Gallego NL, et al. Adjusting pulse amplitude during transcutaneous electrical nerve stimulation (TENS) application produces greater hypoalgesia. J Pain. 2011;12(5):581-90 Available from: http://www.ncbi.nlm.nih.gov/pubmed/21277840. 
33. Cheing GL, Tsui AY, Lo SK, et al. Optimal stimulation duration of TENS in the management of osteoarthritic knee pain. J Rehabil Med. 2003;35(2):62-8.

34. Buhagiar MA, Naylor M, Harris IA, et al. Effect of inpatient rehabilitation vs a monitored home-based program on mobility in patients with total knee arthroplasty. The HIHO randomized clinical trial. JAMA. 2017;317(10):1037-46.

35. Rakel B, Cooper N, Adams HJ, et al. A new transient sham TENS device allows for investigator blinding while delivering a true placebo treatment. J Pain. 2010;11(3):230-8 Available from: http://www.ncbi.nlm.nih.gov/ pubmed/19945354.

36. Farra JT, Young JP, LaMoureauz L. Clinical importance of changes in chronic pain intensity measured on an 11-point numerical pain rating scale. Pain. 2001;94:149-58.

37. Hjermstad MJ, Fayers PM, Haugen DF, et al. Studies comparing numerical rating scales, verbal rating scales, and visual analogue scales for assessment of pain intensity in adults: a systematic literature review. J Pain Symptom Manag. 2011;41(6):1073-93.

38. Gagliese L, Weizblit N, Ellis W, et al. The measurement of postoperative pain: a comparison of intensity scales in younger and older surgical patients. Pain. 2005;117(3):412-20 Available from: http://www.ncbi.nlm.nih.gov/ pubmed/16153776.

39. Scuderi GR, Bourne RB, Noble PC, et al. The new Knee Society Knee Scoring System. Clin Orthop Relat Res. 2012;470(1):3-19 Available from: http://www. ncbi.nlm.nih.gov/pubmed/22045067.

40. Insall JN, Dorr LD, Scott D, et al. Rationale of The Knee Society Clinical Rating System. Clin Orthop Relat Res. 1989:13-4.

41. Gulati A, Pandit $H$, Jenkins $C$, et al. The effect of leg alignment on the outcome of unicompartmental knee replacement. J Bone Jt Surg - Br. 2009; 91-B(4):469-74 Available from: http://www.bjj.boneandjoint.org.uk/cgi/doi/ 10.1302/0301-620X.91B4.22105.

42. Sprague RB. Factors related to extension lag at the knee joint. J Orthop Sport Phys Ther. 1982;3(4):178-82 Available from: http://www.jospt.org/doi/ 10.2519/jospt.1982.3.4.178.

43. Jakobsen TL, Christensen M, Christensen SS, et al. Reliability of knee joint range of motion and circumference measurements after total knee arthroplasty: does tester experience matter? Physiother Res Int. 2010;15:126-34.

44. Le BM, Apap D, Babcock J, et al. The psychometric properties of a modified sit-to-stand test with use of the upper extremities in institutionalized older adults. Percept Mot Skills. 2016;123(1):138-52.

45. Martimbianco ALC, Calabrese FR, Iha LAN, et al. Reliability of the American Knee Society Score (AKSS). Acta Ortop Bras. 2012;20(1):34-8.

46. Dhurve K, Scholes C, El-Tawil S, et al. Multifactorial analysis of dissatisfaction after primary total knee replacement. Knee. 2017;24:856-62.

47. Sun $K$, Xing T, Zhang F, et al. Perioperative transcutaneous electrical acupoint stimulation for postoperative pain relief following laparoscopic surgery: a randomized controlled trial. Clin J Pain. 2016;33(4):340.

48. Schug SA, Palmer GM, Scott DA, et al. Acute pain management: scientific evidence, fourth edition, 2015. Med J Aust. 2016;204(8):315-7 Available from: https://www.mja.com.au/journal/2016/204/8/acute-pain-managementscientific-evidence-fourth-edition-2015.

49. Chughtai M, Elmallah RD, Mistry JB, et al. Nonpharmacologic pain management and muscle strengthening following total knee arthroplasty. J Knee Surg. 2015;29(3):194-200 Available from: http://www.ncbi.nlm.nih.gov/ pubmed/26683980.

50. Sluka KA, Deacon M, Stibal A, et al. Spinal blockade of opioid receptors prevents the analgesia produced by TENS in arthritic rats. J Pharmacol Exp Ther. 1999;289(2):840-6.

51. Liebano RE, Rakel B, Vance CGT, et al. An investigation of the development of analgesic tolerance to TENS in humans. Pain. 2011;152(2):335-42 Available from: http://www.ncbi.nlm.nih.gov/pubmed/21144659.

52. Desantana JM, Santanafilho VJ, Sluka KA. Modulation between high- and lowfrequency transcutaneous electric nerve stimulation delays the development of analgesic tolerance in arthritic rats. Arch Phys Med Rehabil. 2008;89(4):754-60.

53. Chandran P, Sluka KA. Development of opioid tolerance with repeated transcutaneous electrical nerve stimulation administration. Pain. 2003;102(1):195-201.

54. Tokuda M, Tabira K, Masuda T, et al. Effect of modulated-frequency and modulated-intensity transcutaneous electrical nerve stimulation after abdominal surgery. Clin J Pain. 2014;30(7):565-70 Available from: http:// content.wkhealth.com/linkback/openurl?sid=WKPTLP:landingpage\&an= 00002508-201407000-00002.

55. Xiang $\mathrm{XH}$, Chen YM, Zhang JM, et al. Low- and high-frequency transcutaneous electrical acupoint stimulation induces different effects on cerebral $\mu$-opioid receptor availability in rhesus monkeys. J Neurosci Res. 2014;92(5):555-63.

56. Oliveira GAD, Caroline SA, Da SCF, et al. Influence of different frequencies of transcutaneous electrical nerve stimulation on the threshold and pain intensity in young subjects. Einstein. 2014;12(3):318-22.

57. Pitangui ACR, Araújo RC, Bezerra MJS, et al. Low and high-frequency TENS in post-episiotomy pain relief: a randomized, double-blind clinical trial. Braz J Phys Ther. 2014;18(1):72-8.

58. Walsh DM, Howe TE, Johnson Ml, et al. Transcutaneous electrical nerve stimulation for acute pain (Review). Cochrane Database Syst Rev. 2009;(2):CD006142.

59. Johnson MI, Paley CA, Howe TE, Sluka KA. Transcutaneous electrical nerve stimulation for acute pain. Cochrane Database Syst Rev. 2015;Issue 2015(6): CD006142 Available from: http://www.ncbi.nlm.nih.gov/pubmed/26075732.

60. Bjordal JM, Johnson MI, Ljunggreen AE. Transcutaneous electrical nerve stimulation (TENS) can reduce postoperative analgesic consumption. A meta-analysis with assessment of optimal treatment parameters for postoperative pain. Eur J Pain. 2003;7:181-8.

61. Chen $Y$, Zhang $X$, Zhu Y, et al. Systematic review of three electrical stimulation techniques for rehabilitation after total knee arthroplasty. J Arthroplast. 2018;33(7):2330-7 Available from: http://www.ncbi.nlm.nih.gov/ pubmed/29530519.

62. Chen CC, Tabasam G, Johnson MI. Does the pulse frequency of transcutaneous electrical nerve stimulation ( TENS ) influence hypoalgesia? A systematic review of studies using experimental pain and healthy human participants. Physiotherapy. 2008;94:11-20.

63. Bennett Ml, Hughes $\mathrm{N}$, Johnson Ml. Methodological quality in randomised controlled trials of transcutaneous electric nerve stimulation for pain: low fidelity may explain negative findings. Pain. 2011;152(6):1226-32 Available from: http://www.ncbi.nlm.nih.gov/pubmed/21435786.

64. Sluka KA, Bjordal JM, Marchand S, et al. What makes transcutaneous electrical nerve stimulation work? Making sense of the mixed results in the clinical literature. Phys Ther. 2013;93(10):1397-402 Available from: https:// academic.oup.com/ptj/article-lookup/doi/10.2522/ptj.20120281.

65. Beckwee D, Bautmans I, Swinnen E, et al. A systematic review investigating the relationship between efficacy and stimulation parameters when using transcutaneous electrical nerve stimulation after knee arthroplasty. SAGE Open Med. 2014;2:1-13 Available from: http://www.ncbi.nlm.nih.gov/ pubmed/26770730.

66. Desantana JM, Da SL, De Resende MA, et al. Transcutaneous electrical nerve stimulation at both high and low frequencies activates ventrolateral periaqueductal grey to decrease mechanical hyperalgesia in arthritic rats. Neuroscience. 2009;163(4):1233-41.

\section{Ready to submit your research? Choose BMC and benefit from:}

- fast, convenient online submission

- thorough peer review by experienced researchers in your field

- rapid publication on acceptance

- support for research data, including large and complex data types

- gold Open Access which fosters wider collaboration and increased citations

- maximum visibility for your research: over $100 \mathrm{M}$ website views per year

At BMC, research is always in progress.

Learn more biomedcentral.com/submissions 